\title{
RESEARCH
}

Open Access

\section{Human placenta-derived mesenchymal stem cells stimulate ovarian function via miR-145 and bone morphogenetic protein signaling in aged rats}

Kyeoung-Hwa Kim¹, Eun-Young Kim', Gi Jin Kim', Jung-Jae Ko ${ }^{1}$, Kwang Yul Cha², Mi Kyung Koong ${ }^{3}$ and Kyung-Ah Lee ${ }^{1 *}$

\begin{abstract}
Background: Aging has detrimental effects on the ovary, such as a progressive reduction in fertility and decreased hormone production, that greatly reduce the quality of life of women. Thus, the current study was undertaken to investigate whether human placenta-derived mesenchymal stem cell (hPD-MSC) treatment can restore the decreases in folliculogenesis and ovarian function that occur with aging.

Methods: Acclimatized 52-week-old female SD rats were randomly divided into four groups: single hPD-MSC $(5 \times$ $10^{5}$ ) therapy, multiple (three times, 10-day intervals) hPD-MSC therapy, control (PBS), and non-treated groups. hPDMSC therapy was conducted by tail vein injection into aged rats. The rats were sacrificed 1, 2, 3, and 5 weeks after the last injection. hPD-MSC tracking and follicle numbers were histologically confirmed. The serum levels of sex hormones and circulating miRNAs were detected by ELISA and qRT-PCR, respectively. TGF- $\beta$ superfamily proteins and SMAD proteins in the ovary were detected by Western blot analysis.

Results: We observed that multiple transplantations of hPD-MSCs more effectively promoted primordial follicle activation and ovarian hormone ( $E_{2}$ and $\left.A M H\right)$ production than a single injection. After hPD-MSC therapy, the levels of miR-21-5p, miR132-3p, and miR-212-3p, miRNAs associated with the ovarian reserve, were increased in the serum. Moreover, miRNAs (miR16-5p, miR-34a-5p, and miR-191-5p) with known adverse effects on folliculogenesis were markedly suppressed. Importantly, the level of miR-145-5p was reduced after single- or multiple-injection hPD-MSC therapy, and we confirmed that miR-145-5p targets Bmpr2 but not Tgfor2. Interestingly, downregulation of miR-145-5p led to an increase in BMPR2, and activation of SMAD signaling concurrently increased primordial follicle development and the number of primary and antral follicles.

Conclusions: Our study verified that multiple intravenous injections of hPD-MSCs led to improved ovarian function via miR145-5p and BMP-SMAD signaling and proposed the future therapeutic potential of hPD-MSCs to promote ovarian function in women at advanced age to improve their quality of life during climacterium.
\end{abstract}

Keywords: Aging, Stem cell therapy, Hormone biosynthesis, miR-145, Primordial follicle activation, Follicular development

\footnotetext{
* Correspondence: leeka@cha.ac.kr

'Department of Biomedical Science, Institute of Reproductive Medicine,

College of Life Science, CHA University, Pangyo-Ro 335, Bundang-gu,

Seongnam-si, Gyeonggi-do 13488, South Korea

Full list of author information is available at the end of the article
}

C C The Author(s). 2020 Open Access This article is licensed under a Creative Commons Attribution 4.0 International License, which permits use, sharing, adaptation, distribution and reproduction in any medium or format, as long as you give appropriate credit to the original author(s) and the source, provide a link to the Creative Commons licence, and indicate if changes were made. The images or other third party material in this article are included in the article's Creative Commons licence, unless indicated otherwise in a credit line to the material. If material is not included in the article's Creative Commons licence and your intended use is not permitted by statutory regulation or exceeds the permitted use, you will need to obtain permission directly from the copyright holder. To view a copy of this licence, visit http://creativecommons.org/licenses/by/4.0/ The Creative Commons Public Domain Dedication waiver (http://creativecommons.org/publicdomain/zero/1.0/) applies to the data made available in this article, unless otherwise stated in a credit line to the data. 


\section{Background}

The ovaries are the main female reproductive organs that sustain reproductive system health and safeguard the quality of life of women through hormone biosynthesis. However, the aging of the ovaries occurs faster than that of other reproductive organs, such as the uterus and pituitary gland [1]. Ovarian aging is accompanied by a significant decline in the number of primordial follicles, an ovarian reservoir, and an increased number of low-quality oocytes, which contribute to the gradual decline in fertility and thus the increase in the incidence of natural sterility caused by menopause [2, 3]. Additionally, menopause is the final step in the process referred to as ovarian aging [3]. Aging-associated menopause leads to systemic complications such as menopausal symptoms, metabolic syndrome, dementia, osteoporosis, and heart disease $[4,5]$. Female reproductive aging is an increasing public health issue in modern society in which women pursue a career long before marriage at more than 30 years of age.

A primordial follicle consists of an immature oocyte arrested at the diplotene stage of meiosis I surrounded by several flattened pregranulosa cells [6]. At birth, the fetal ovary usually contains approximately 300,000 to 400,000 primordial follicles, and this number declines with age [7, 8]. At the beginning of puberty, quiescent primordial follicles develop into primary follicles under the control of AKT and MTOR signaling induced by stimulation factors [9]. These factors include kit ligand, VEGF, BMP4, BMP7, LIF, FGF2 (known as basic FGF), and others [10-15]. However, most follicles undergo apoptosis, a process known as atresia, and only 300 to 400 follicles are selected for ovulation during the reproductive lifetime of an adult female [16]. Therefore, the pool of primordial follicles in the ovarian reserve is the major determinant of the reproductive period, healthy ovarian lifespan, and thus the fertility and reproductive health of an individual [17].

Circulating microRNAs (miRNAs) are small noncoding RNA molecules that negatively regulate gene expression through mRNA cleavage, translational repression, or mRNA degradation [18]. It has also been shown that circulating miRNAs in serum or plasma can modulate gene expression during aging processes and can affect aging phenotypes [19]. Thus, circulating miRNAs have recently emerged as important regulators of aging and frailty in elderly individuals. In addition, several studies have identified circulating miRNA populations specifically associated with ovarian function, such as follicle development, ovulation, granulosa cell apoptosis, and steroidogenesis [20, 21]. Therefore, ovarian function-related and/or ovarian-specific circulating miRNAs detected in the serum can be used as early diagnostic and prognostic monitoring markers in ovarian dysfunction with aging.

In previous studies, human placenta-derived mesenchymal stem cell (hPD-MSC) transplantation was shown to have positive effects on degenerative diseases, such as antifibrosis, anti-inflammation, and antiapoptosis effects [2224]. Moreover, transplantation of hPD-MSCs had therapeutic effects on young ovariectomized (Ovx) rats by increasing the levels of $E_{2}$ and folliculogenesis-related gene expression [25]. However, whether hPD-MSC therapy can restore ovarian follicular development and the other functions of naturally aged ovaries is still unknown. Thus, the aims of this study were to determine whether hPD-MSC therapy could counteract age-related ovarian dysfunctions and decrease ovarian reserve in advanced-age rat models and to evaluate the efficacy of single-injection hPD-MSC therapy and multiple-injection hPD-MSC therapy on ageassociated physiological characteristics, including ovarian functions, in old female rats.

\section{Methods \\ Reagents}

Chemicals and reagents were obtained from SigmaAldrich (MO, USA) unless otherwise noted.

\section{Experimental design}

All experiments involving animals were approved by the Institutional Animal Care and Use Committee (IACUC 190163) of the CHA Laboratory Animal Research Center. Sprague-Dawley (SD) rats were provided by Janvier Labs (Le Genest-Saint-Isle, France) and maintained at the breeding facility at the CHA Bio Complex of CHA University. SD rats (52-54 weeks of age) were used for all experiments. These animals were randomly divided into singleand multiple-injection hPD-MSC therapy groups $(n=24$ each group), a control group, and an untreated group. For single-injection hPD-MSC therapy, $5 \times 10^{5}$ hPD-MSCs were suspended in $0.2 \mathrm{ml}$ of phosphate-buffered saline (PBS) and injected into rats via the tail vein. For multipleinjection hPD-MSC therapy, a series of PKH67-labeled hPD-MSCs were injected three times at 10-day intervals or 4-week intervals. The rats from the control group were only injected with an equal volume of PBS. The rats were sacrificed 1, 2, 3, and 5 weeks following the transplantation of hPD-MSCs, and the organs and blood samples were collected and immediately frozen. Ovaries were fixed with $4 \%$ paraformaldehyde or optimal cutting temperature (OCT) compound (Leica Biosystems, IL, USA) for further processing. Serum was collected by centrifugation and stored at $-80^{\circ} \mathrm{C}$ for hormone assays.

\section{Culture of hPD-MSCs}

hPD-MSCs were kindly provided by Dr. Gi Jin Kim. hPDMSCs were isolated and characterized as described previously $[22,25]$. Placentas ( $38 \pm 2$ gestational weeks) were collected from women who were free of any medical, obstetrical, or surgical complications. All participants provided written informed consent prior to placenta collection. 
hPD-MSCs were isolated from human placental chorionic plates and approved by the Institutional Review Board of CHA General Hospital, Seoul, Korea (IRB 07-18). Briefly, the fetal membrane was removed from the chorionic plate of each placenta, and cells were treated with $0.5 \%$ collagenase IV (Sigma-Aldrich) for $30 \mathrm{~min}$ at $37^{\circ} \mathrm{C}$. The harvested cells were plated $\left(2 \times 10^{5}\right.$ cells $\left./ \mathrm{cm}^{2}\right)$ with $\alpha$-MEM supplemented with $10 \%$ fetal bovine serum (FBS; Corning, NY, USA), $1 \%$ penicillin-streptomycin (Life Technologies, USA), $1 \mathrm{mg} / \mathrm{ml}$ heparin (Sigma-Aldrich), and $100 \mu \mathrm{g} / \mathrm{ml} \mathrm{FGF4}$ (PeproTech, NJ, USA). The cells were maintained in a humidified atmosphere containing $5 \% \mathrm{CO}_{2}$ and grown until 80-90\% confluence.

\section{Labeling and tracking of hPD-MSCs}

To track and locate the transplanted hPD-MSCs in the ovarian tissues, the cells were prelabeled with PKH67 Green Fluorescent Cell Linker kits (Sigma-Aldrich) according to the manufacturer's instructions. Briefly, a total of $2 \times 10^{7}$ hPD-MSCs were washed and gently resuspended in $1 \mathrm{ml}$ of Diluent C. In parallel, $4 \mu \mathrm{l}$ of PKH67 dye was added to $1 \mathrm{ml}$ of Diluent C $\left(4 \times 10^{-6} \mathrm{M}\right)$ and incubated with the hPD-MSC solution for $5 \mathrm{~min}$. To bind excess dye, the same volume of $1 \%$ BSA was added. The labeled hPD-MSCs were washed and observed by fluorescence microscopy.

PKH67-labeled hPD-MSCs were transplanted into rats via the tail vein. Ovaries were fixed with OCT compound and made into fresh sections (12 $\mu \mathrm{m}$ thick). After fixation with $4 \%$ paraformaldehyde for $20 \mathrm{~min}$, the sections were washed and incubated with 2-(4-amidinophenyl)-6-indolecarbamidine dihydrochloride (DAPI; SigmaAldrich) at room temperature for $10 \mathrm{~min}$. The sections were then imaged under a laser scanning confocal microscope (LSCM; Leica, Wetzlar, Germany).

\section{Genome extraction}

Rat genomic DNA was isolated from the ovary, liver, and lung after hPD-MSC therapy as described previously [26]. After RNA extraction with $500 \mu \mathrm{l}$ of TRIzol (Invitrogen, CA, USA), we added $250 \mu$ of back extraction buffer (BEB; $4 \mathrm{M}$ guanidine thiocyanate; $50 \mathrm{mM}$ sodium citrate; $1 \mathrm{M}$ Tris, $\mathrm{pH}$ 8.0) and allowed the interphaseorganic phase mixtures to sit at room temperature for $10 \mathrm{~min}$. The samples were then centrifuged at 13,000 $\mathrm{rpm}$ for $15 \mathrm{~min}$ at $4{ }^{\circ} \mathrm{C}$. The upper phase was removed, an equal volume of $100 \%$ isopropanol was added, and the samples were incubated overnight at $-80^{\circ} \mathrm{C}$. After incubation, the samples were centrifuged again at $13,000 \mathrm{rpm}$ for $15 \mathrm{~min}$ at $4{ }^{\circ} \mathrm{C}$. The supernatant was removed, and the pellets were washed 3 times with $70 \%$ ethanol. The samples were eluted in a final volume of $30 \mu \mathrm{l}$ of Tris-EDTA $(10 \mathrm{mM}$ Tris; $0.1 \mathrm{mM}$ EDTA, pH 8.0).

\section{Human Alu sequence and RT-PCR}

We synthesized the primers as described previously by Walker et al. [27]. The primer sequences were as follows: forward primer (AluYb8_F; position 48-69), 5'CGAGGCGGGTGGATCATGAGGT-3'; reverse primer (AluYb8_R; position 273-254), 5'-TCTGTCGCCC AGGCCGGACT-3'.

The extracted genomic DNA was quantified using a NanoDrop UV spectrophotometer (Thermo Fisher Scientific, MA, USA). RT-PCR was performed with $A l u Y b 8$ primers and $100 \mathrm{ng}$ of genomic DNA from the various organs after single-injection hPD-MSC therapy. RT-PCR products were separated by electrophoresis on a $1.5 \%$ agarose gel and analyzed using a Gel Doc ${ }^{\text {Th }}$ EZ Imager (Bio-Rad, CA, USA).

\section{Ovarian morphology and follicle counting}

To analyze ovarian morphology and follicle counts, six ovaries from each group were collected at the indicated time points $(1,2,3$, and 5 weeks after single- or multiple-injection hPD-MSC therapy) and fixed with $4 \%$ paraformaldehyde for $48 \mathrm{~h}$ at $4{ }^{\circ} \mathrm{C}$. The ovaries were dehydrated, embedded in paraffin, and serially cut into $7-\mu \mathrm{m}$ sections. After hematoxylin and eosin (H\&E) staining, sections were observed and imaged by an optical microscope (Nikon Corporation, Tokyo, Japan). Each follicle stage, namely primordial, primary, secondary, preantral, and antral follicle stages, was counted in every tenth section through the ovary.

\section{Circulating miRNA extraction}

Circulating miRNAs were extracted from $200 \mu \mathrm{l}$ of serum using an miRNeasy serum/plasma kit (Qiagen, Hilden, Germany) according to the manufacturers' instructions. Briefly, serum samples were lysed with $1 \mathrm{ml}$ of Qiazol and spiked with $3.5 \mu$ of synthetic cel-miR-39 mimic $\left(1.6 \times 10^{8}\right.$ copies/ $\mu$ l; Qiagen $)$ to test extraction efficiency. Chloroform $(200 \mu \mathrm{l})$ was used for phase separation at $4{ }^{\circ} \mathrm{C}$ and $10,000 \mathrm{rpm}$. The aqueous phase $(600 \mu \mathrm{l})$ was added to $900 \mu \mathrm{l}$ of $100 \%$ ethanol (Merck Millipore, MA, USA) and subsequently transferred into an RNeasy MiniElute spin column. These columns were washed with RWT buffer, RPE buffer, and $80 \%$ ethanol (Merck Millipore). All samples were eluted in $14 \mu \mathrm{l}$ of RNase-free water.

\section{Quantitative real-time RT-PCR (qRT-PCR)}

For reverse transcription (HB_I RT Reaction kit; Heimbiotek, Pangyo, Korea), $11 \mu \mathrm{l}$ of the eluted miRNAs was mixed with $2 \mu \mathrm{l}$ of miR enzyme mix, $2 \mu \mathrm{l}$ of miR multi buffer, and $5 \mu \mathrm{l}$ of nucleic mix I. The samples were incubated for $60 \mathrm{~min}$ at $37^{\circ} \mathrm{C}$ and for $5 \mathrm{~min}$ at $95^{\circ} \mathrm{C}$.

To measure the amounts of circulating miRNAs in serum after hPD-MSC therapy, quantitative real-time 
RT-PCR analysis was performed using a CFX96 Touch $^{\text {Tx }}$ Real-Time PCR Detection System (Bio-Rad). The primers used to amplify the selected miRNAs were purchased from Heimbiotek (Nucleic Mix II). The HB miR multi assay kit system I (Heimbiotek) was used for monitoring amplification, and the results were evaluated using CFX Maestro software (Bio-Rad). Melting curves were used to identify nonspecific amplification products. The expression of each miRNA was normalized to the expression of cel-miR-39. The relative target miRNA expression was calculated using the comparative $\mathrm{C}_{\mathrm{T}}$ method.

\section{Western blotting}

To confirm protein expression, Western blotting was performed as previously described [28]. Briefly, a protein extract was separated using $10 \%$ or $12 \%$ SDS-PAGE and transferred onto a PVDF membrane (Amersham Biosciences, Piscataway, NJ). After blocking, the membrane was incubated with antibodies against BMPR2 (1:1000; ab96826, Abcam, Cambridge, MA), TGFBR2 (1:1000; \#79424, Cell Signaling Technology, Danvers, MA), ACVR1B (1:1000; ab109300, Abcam), ACVR2A (1:1000; ab96793, Abcam), SMAD1 (1:1000; \#9743, Cell Signaling Technology), phospho-SMAD1/5 (p-SMAD1/5; 1:1000; \#9516, Cell Signaling Technology), SMAD3 (1:1000; \#9513, Cell Signaling Technology), phospho-SMAD3 (pSMAD3; 1:1000; \#9520, Cell Signaling Technology), and GAPDH (1:2000; sc-47,724, Santa Cruz Biotechnology, Dallas, TX), followed by incubation with HRP-conjugated anti-rabbit IgG (1:5000; \#7074, Cell Signaling Technology, Danvers, MA, USA) or anti-mouse IgG (1:2000; A2554). Bound antibodies were detected using an enhanced chemiluminescence detection system (Amersham Biosciences) according to the manufacturer's instructions.

\section{Levels of $E_{2}$ and $A M H$ in serum}

One sample of $2 \mathrm{ml}$ of blood was collected from each rat. The blood samples were centrifuged, and the serum was separated into two parts: one part was used for AMH analysis, and one part was used for $E_{2}$ analysis. The serum levels of $A M H$ and $E_{2}$ at the indicated time points $(1,2,3$, and 5 weeks after single- or multiple-injection hPD-MSC therapy) were measured using the Elecsys ${ }^{\circ} \mathrm{AMH}$ immunoassay (Roche Diagnostics $\mathrm{GmbH}$, Mannheim, Germany) and Elecsys ${ }^{\circ}$ Estradiol III (Roche Diagnostics GmbH), respectively. All serum markers $\left(\mathrm{AMH}\right.$ and $\mathrm{E}_{2}$ ) were determined in a single measurement on the e601 module of the fully automated Cobas 6000 system (Roche Diagnostics $\mathrm{GmbH}$ ). Assays were performed according to the manufacturer's instructions.

\section{Statistical analysis}

Data are expressed as the mean \pm SEM. Statistical analyses were performed using Student's $t$ test for paired samples (non-treated group vs. single-injection hPDMSC therapy group; control group vs. multiple-injection hPD-MSC therapy group), and $p<0.05$ was considered statistically significant.

\section{Results}

hPD-MSCs were detected in ovaries after cell therapy

To track and locate hPD-MSCs in the ovary, the cells were prelabeled with PKH67 before injection. The staining of hPD-MSCs with PKH67 revealed consistent, clear, and uniform distribution of the labeled cell membrane as observed using an inverted microscope (Fig. 1a). The location and fate of injected PKH67-labeled hPD-MSCs in ovarian tissues were tracked at 1 week, 2 weeks, 3 weeks, and 5 weeks after tail vein injection (Fig. 1b). The results show that PKH67-labeled hPD-MSCs were located in the interstitium and surrounding the follicles (white arrowheads) of ovaries after multiple-injection therapy. We found that PKH67labeled hPD-MSCs were observed in the therapy groups, starting on the first week after multiple cell injections via the tail vein (Fig. 1b (b1-3)). In addition, a green fluorescent signal could still be observed in ovaries at 5 weeks after the multiple-injection therapy (Fig. 1b (h1-3)). However, the green fluorescent signal gradually decreased with time (Fig. 1b). On the other hand, human DNA (AluYb8) sequences were found after single-injection therapy by tail vein injection. We detected the PCR products of AluYb8 sequences in ovaries 2-3 weeks after single-injection therapy (Additional file 1: Figure S1). These results indicate that hPD-MSCs were detected faster in ovaries and showed long-term maintenance after multiple hPD-MSC injections than after a single injection.

\section{hPD-MSC therapy induced follicular development and steroid production}

To explore the effects of hPD-MSC therapy on the decreased ovarian reserve induced by aging, histological analysis was performed. For this study, follicles have been classified according to their morphological characteristics acquired during follicular development, including the diameter, oocyte diameter, and the granulosa cell number and morphology. A primordial follicle was defined as a small oocyte surrounded by flattened pregranulosa cells; a primary follicle was defined as having a larger oocyte surrounded by one layer of cuboidal and proliferative granulosa cells; secondary and preantral follicles were defined as follicles with a larger diameter surrounded by two or more layers of granulosa cells with no antrum; and an antral follicle was characterized by granulosa cells with more than one small antral cavity or with one large antrum (Fig. 2a). Histological studies of the ovaries found no significant difference in the number of primordial and primary follicles after single-injection therapy (Fig. 2b, c). However, there were significant increases in the numbers of secondary, 


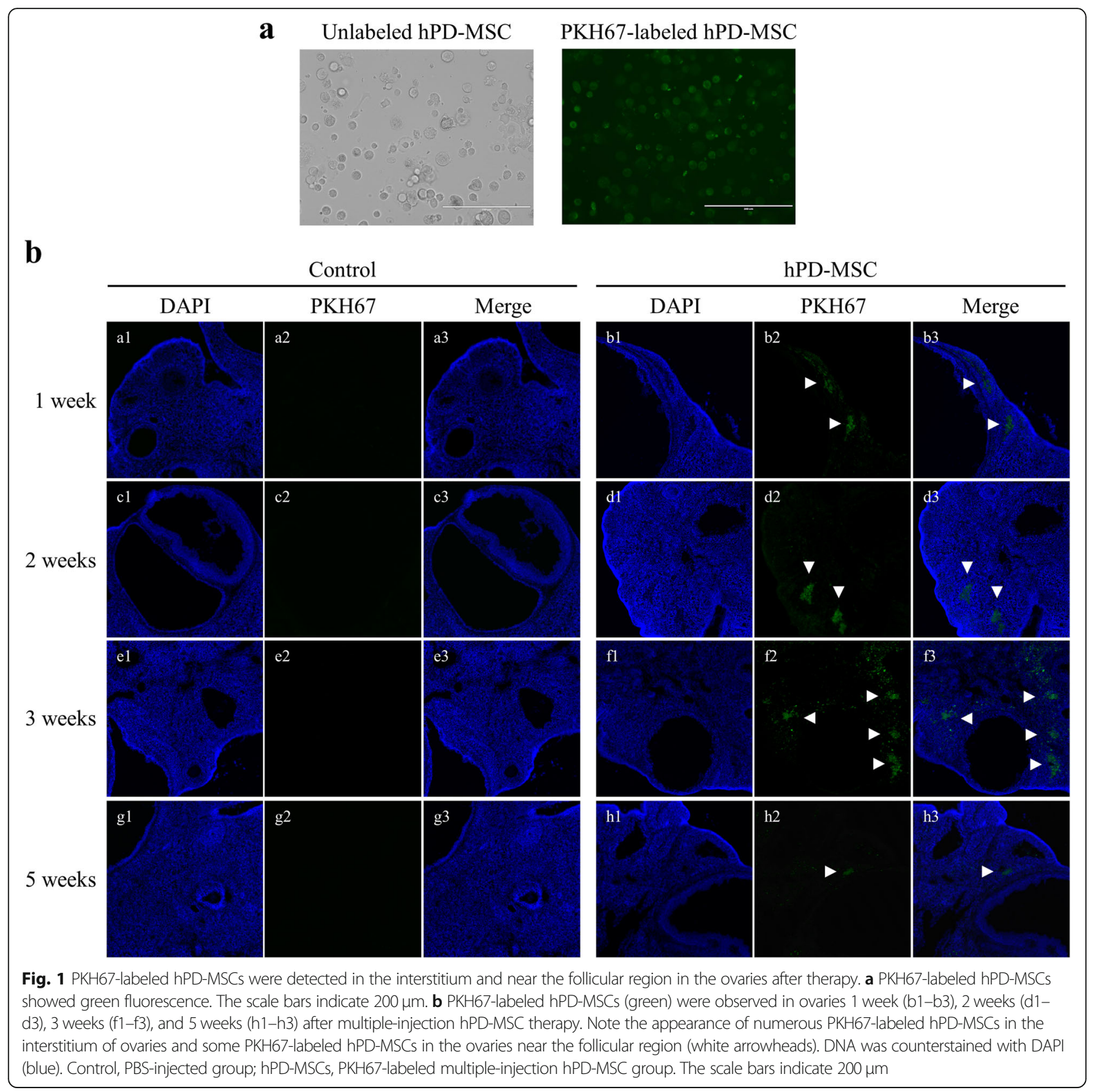

preantral, and antral follicles at 1 week after singleinjection hPD-MSC therapy compared with the control (Fig. 2b, c; gray striped bar vs. gray bar). These changes were improved by cell transplantation in the singleinjection therapy group. Surprisingly, the number of primary follicles substantially increased at 2 weeks and 3 weeks after tail vein injection in the multiple-injection therapy group compared with the control group (Fig. 2b, c; 2 weeks, orange striped bar vs. orange bar; 3 weeks, green striped bar vs. green bar). These results appear to represent the strong positive effects of the multipleinjection therapy on early folliculogenesis. We observed a more than 2-fold increase in the number of primary follicles following multiple-injection hPD-MSC therapy compared to that following single-injection therapy (Fig. 2c). Taken together, these results demonstrate that both the single- and multiple-injection therapies can rescue the ovarian reserve and follicle development and that multiple-injection hPD-MSC therapy was more effective for primordial follicle activation and subsequent follicular development than a single injection.

Unexpectedly, we found that the number of primordial follicles was significantly increased 2 weeks after multipleinjection therapy compared with the control group (Fig. 2c; 


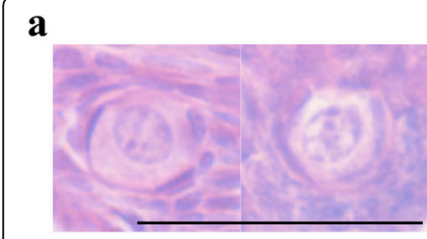

Primordial follicle

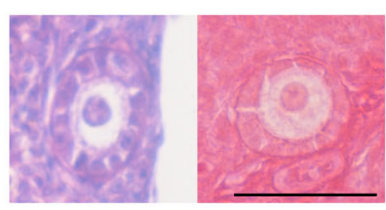

Primary follicle

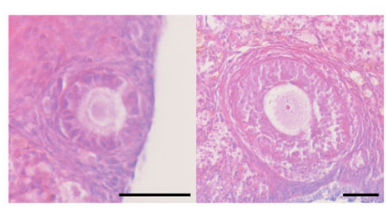

Secondary and preantral follicle

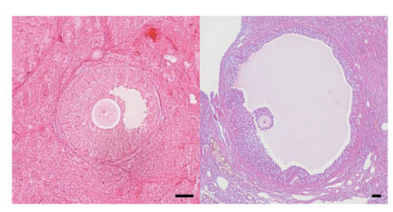

Antral follicle

b
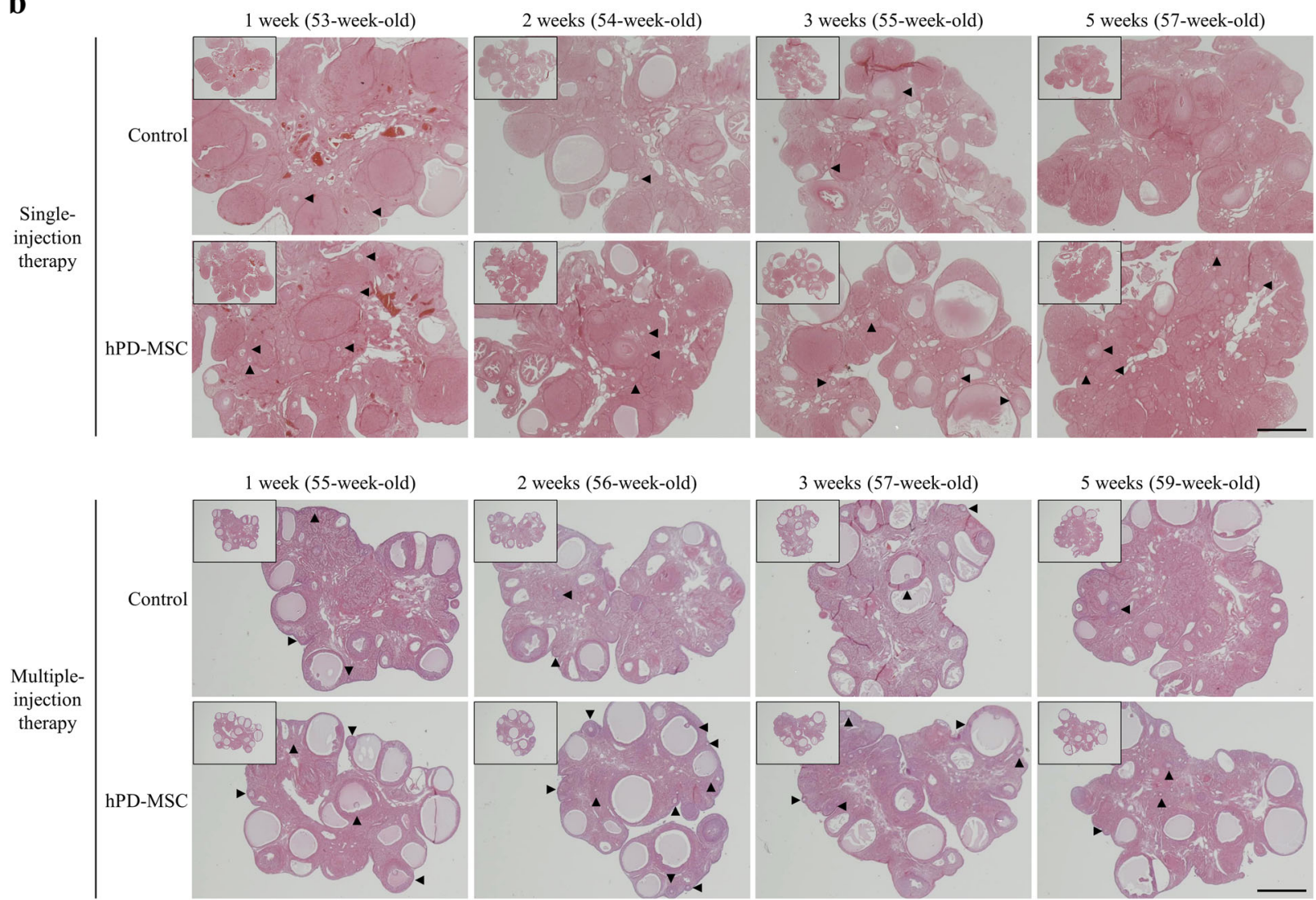

c

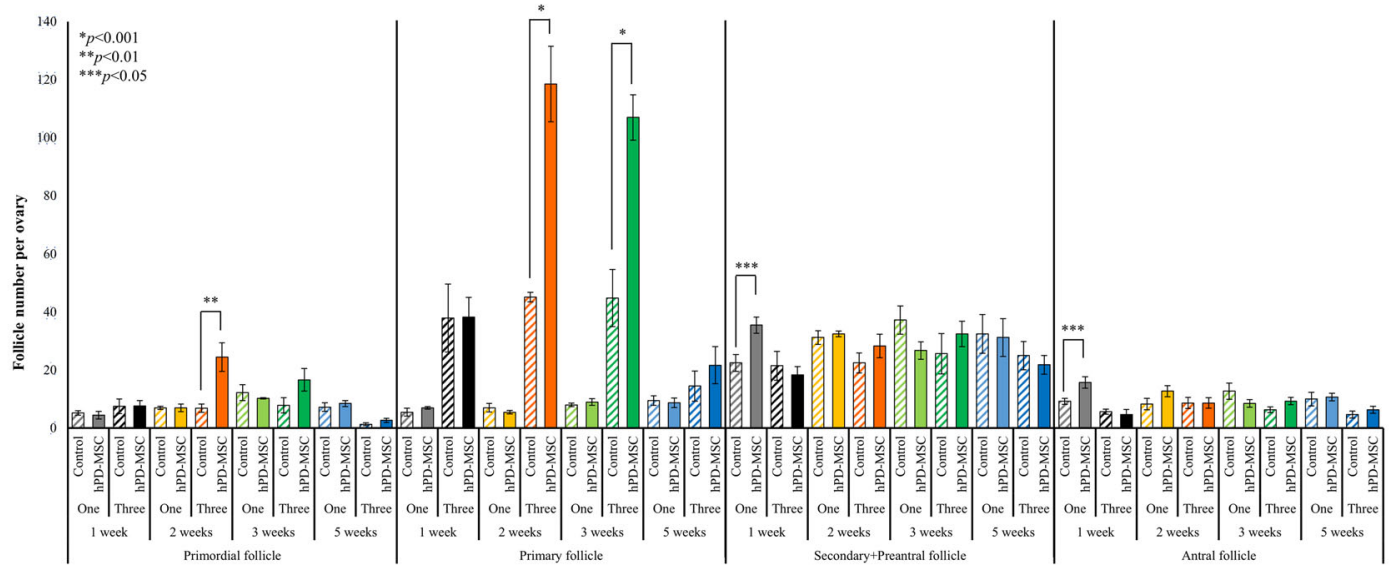

Fig. 2 (See legend on next page.) 
(See figure on previous page.)

Fig. 2 hPD-MSC therapy attenuates ovarian dysfunction by inducing follicular development. a Classification of the ovarian follicular stages used for analysis. The scale bars indicate $50 \mu \mathrm{m}$. b Ovarian structure after hPD-MSC therapy. Six rats in each group were randomly selected at the indicated time points following hPD-MSC injection. By histological analyses, serially sectioned whole ovaries were stained with $\mathrm{H \& E}$, and follicles at different stages were counted in the control and hPD-MSC therapy groups. Black arrowheads indicate ovarian follicles. Scale bars indicate 1 mm. c After single- or multiple-injection therapy, the numbers of follicles at various stages were counted and compared with the numbers in the control group. hPD-MSC therapy resulted in a significant increase in the number of developmental follicles. The results are presented as the mean \pm SEM, and statistical $p$ values were calculated; ${ }^{*} p<0.001$, ${ }^{* *} p<0.01$, and ${ }^{* * *} p<0.05$. One, single-injection hPD-MSC therapy; Three, multiple-injection hPD-MSC therapy

primordial follicle, 2 weeks, orange striped bar vs. orange bar). We thought that this increased number of primordial follicles in ovaries after hPD-MSC injection was a coincidence rather than an effect of the multiple-injection therapy because the total number of primordial follicles is fixed at birth, and a rat with a larger initial ovarian reservoir was assigned to that group despite randomly dividing the rats.

To determine whether single-injection hPD-MSC therapy or multiple-injection hPD-MSC therapies could improve ovarian dysfunction with aging, the levels of $E_{2}$ and $\mathrm{AMH}$ in serum were measured. We observed that $E_{2}$ levels were not significantly different between the control and single-injection therapy groups (Fig. 3a); however, we found that $E_{2}$ levels were significantly increased at 5 weeks after multiple-injection hPD-MSC therapy (Fig. 3b). As shown in Fig. 3c, the levels of AMH in the control group were gradually reduced over time. The levels of AMH in the cell therapy group slightly decreased over weeks 1-2 but increased over weeks 3-5 after therapy, showing a sustained profile (Fig. 3c). Moreover, the levels of AMH were markedly increased in the single-injection cell therapy groups at 5 weeks compared to the control group (Fig. 3c). Because the first sampling time point for the multiple-injection therapy group was 20 days (approximately 3 weeks) after than that for the single-injection therapy group, the level of AMH was relatively low in the multiple-injection therapy groups (Fig. 3d). Furthermore, we observed that AMH levels were gradually reduced in the control group over time, while after multiple-injection hPD-MSC therapy, we found that AMH levels were significantly increased (Fig. 3d). These results imply that hPD-MSC therapy may enhance AMH secretion by promoting granulosa cell proliferation and regulating follicle development. Interestingly, AMH levels increased at a faster rate in serum in the multiple-injection hPD-MSC therapy group than in the single-injection therapy group, with the multiple-injection hPD-MSC therapy group showing higher AMH levels 2-3 weeks after injection (Fig. 3c, d). Thus, the multiple-injection therapy was significantly more effective in sustaining the $\mathrm{E}_{2}$ and $\mathrm{AMH}$ levels than the single-injection therapy. These results indicate that hPD-MSC therapy can improve follicular development and concurrently the aging-related decline in $E_{2}$ and AMH hormone production.
The multiple-injection therapy, three injections of hPDMSCs at 10-day intervals, stimulated early follicular development and steroid biosynthesis in aging ovaries. In contrast, compared to the control group, three injections of hPD-MSCs at 4-week intervals did not significantly affect either the numbers of follicles (Additional file 2: Figure S2a) or the levels of AMH (Additional file 2: Figure S2c). The findings showed that $\mathrm{E}_{2}$ levels were significantly reduced 1 week after three injections of hPD-MSCs at 4week intervals, but thereafter, the $\mathrm{E}_{2}$ levels of this group were not significantly different from those of the control group (Additional file 2: Figure S2b). These results suggested that the multiple-injection therapy at 10-day intervals improved ovarian function by follicle development, whereas the multiple-injection therapy at 4-week intervals was not effective. Thus, longer injection intervals for multiple-injection therapy did not show therapeutic effects on follicle development and ovarian hormone production. Because rats have shorter lifespans than humans, 10 rat days is comparable to one human year $[29,30]$. The differences in the results between the multiple-injection therapies with short and long intervals in this rat study may provide useful information for designing future clinical tests with older women.

\section{Changes in circulating miRNAs after hPD-MSC therapy reflect the ovarian reserve and stimulate ovarian function} The altered expression of miRNAs affects folliculogenesis and ovarian steroidogenesis [20, 21, 31]. Because hPDMSC therapy improved ovarian aging phenotypes, we evaluated whether circulating miRNAs could be used as potential biomarkers of the ovarian reserve and ovarian health after hPD-MSC therapy in aged female rats. Seven circulating miRNAs were chosen for further evaluation based on searching the MEDLINE database through PubMed for evidence-based literature associated with ovarian aging with keywords, such as primordial follicle initiation, follicle development, hormone production, and granulosa cell apoptosis [32-37]. The expression of rnomiR16-5p, miR-21-5p, miR-34a-5p, miR-132-3p, miR-145$5 p$, miR-191-5p, and miR-212-3p in serum was analyzed and normalized to the expression of cel-miR-39. Among the selected circulating miRNAs, miR-21-5p, miR-132-3p, and miR-212-3p were upregulated in the hPD-MSC 
$\mathbf{a}$

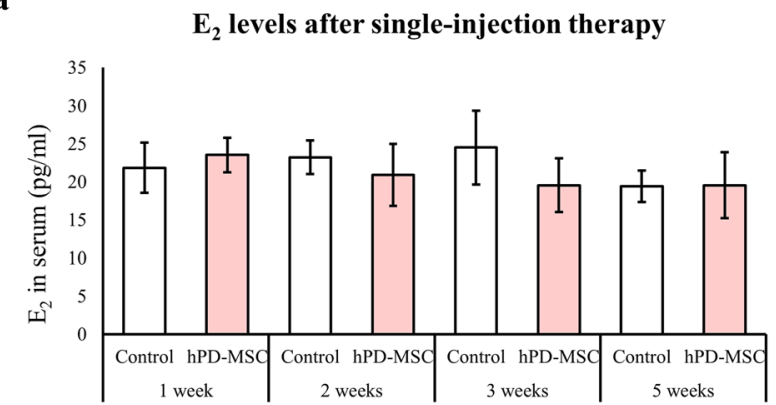

c

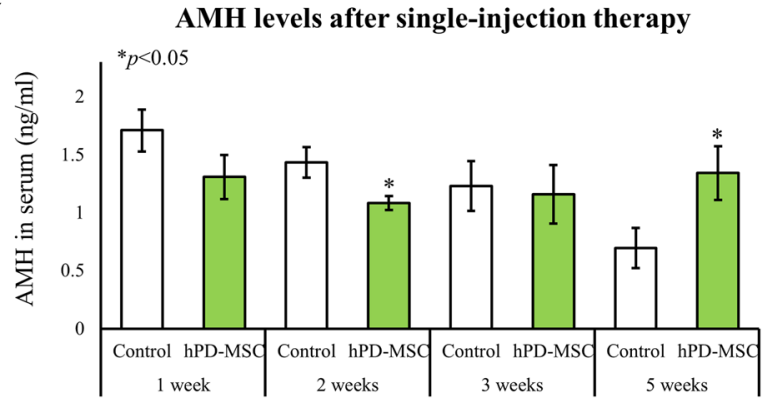

b

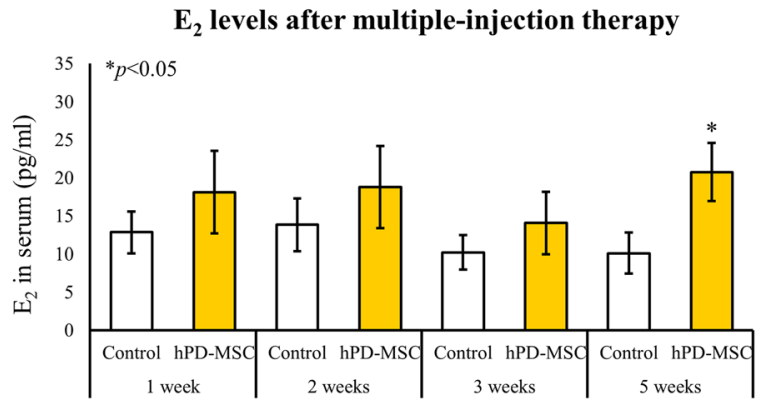

d

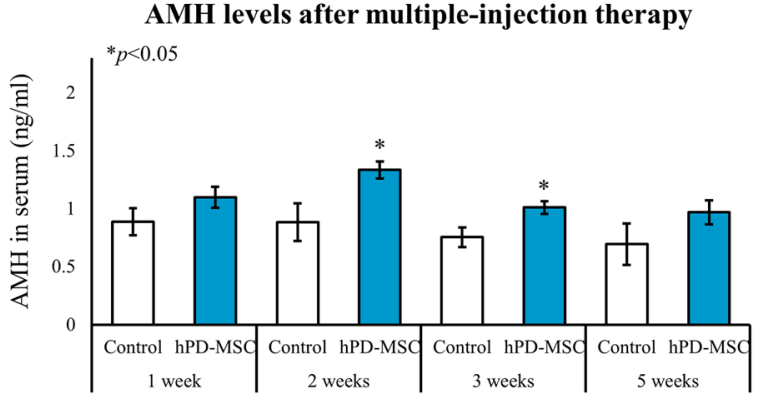

Fig. 3 hPD-MSC therapy improves serum levels of $E_{2}$ and $A M H$ in aged rats. Levels of $E_{2}(\mathbf{a}, \mathbf{b})$ and $A M H(\mathbf{c}, \mathbf{d})$ as determined by ELISA at various time points after single- or multiple-injection hPD-MSC therapy. Data are presented as the mean \pm SEM. The asterisk represents statistical significance at $p<0.05$

therapy groups compared with the control group (Fig. 4a), indicating that they could be used to distinguish the aging control groups from the healthier therapy groups. Moreover, the expression of miR-16-5p, miR-34a-5p, miR-145$5 \mathrm{p}$, and miR-191-5p, related to the suppression of primordial follicle initiation and folliculogenesis, was downregulated in the hPD-MSC therapy groups compared with the control group (Fig. 4b).

\section{Downregulation of miR-145 induces primordial follicle activation by BMPs and the SMAD signaling pathway}

We verified that hPD-MSC therapy led to changes in circulating miRNAs involved in the ovarian reserve. We noted that the expression of miR-145-5p was significantly suppressed after single-injection therapy (Fig. 4b). Yang et al. [32] reported that the miR-145 signaling pathway is involved in the initiation of primordial follicle development. Therefore, we examined the expression levels of circulating miR-145-5p after multiple-injection therapy, and as shown in Fig. 5a, the levels of circulating miR-145-5p were markedly decreased in the serum of animals in the cell therapy groups compared with that in the control group at 2 weeks and 3 weeks, similar to the effects of single-injection hPD-MSC therapy.

The transforming growth factor beta (TGF- $\beta$ ) superfamily has been implicated to promote primordial follicle activation [38]. Using numerous computational algorithms, such as TargetScan, miRnada, miRmap, and miRWork, we found that the putative miR-145 targets TGF- $\beta$ family members and many well-known factors in follicular development, such as Acvr1b, Acvr2a, Bmpr2, Tgfbr2, Smad1, and Smad3. The TGF- $\beta$ superfamily is broadly divided into two major classes: (1) TGF- $\beta \mathrm{s}$, Activins, Myostatin, and Nodal, which act via SMAD2 and SMAD3, and (2) BMPs, GDFs, and AMH, which act via SMAD1, SMAD5, and SMAD8 [39]. TGF- $\beta$ family ligands bind to receptor serine kinases that phosphorylate SMAD proteins, which form transcriptional complexes that regulate specific genes (Fig. 5b). Thus, we analyzed whether the downregulation of circulating miR-145-5p caused by multiple-injection therapy affected the expression of these putative target proteins in ovaries identified by computational algorithms. As shown in Fig. 5c, the expression level of the TGFBR2 protein was relatively high after multiple-injection therapy. However, the expression of p-SMAD3, which is downstream of TGF- $\beta$, was not detected in ovaries with or without hPD-MSC stimulation, indicating that TGF- $\beta$ signaling is not involved in primordial follicle activation (Figs. $5 \mathrm{c}$ and 6). The protein expression of ACVR2A was markedly upregulated, whereas no change in ACVR1B protein expression was observed in the multiple-injection hPD-MSC therapy group compared with the control group (Fig. 5c). The BMP receptor and SMAD1/5/8 are downstream targets of BMP signaling in a positive feedback loop. Enhanced BMPR2 protein expression was observed in ovaries 2-5 weeks after multiple-injection hPD-MSC therapy (Fig. 5c). 


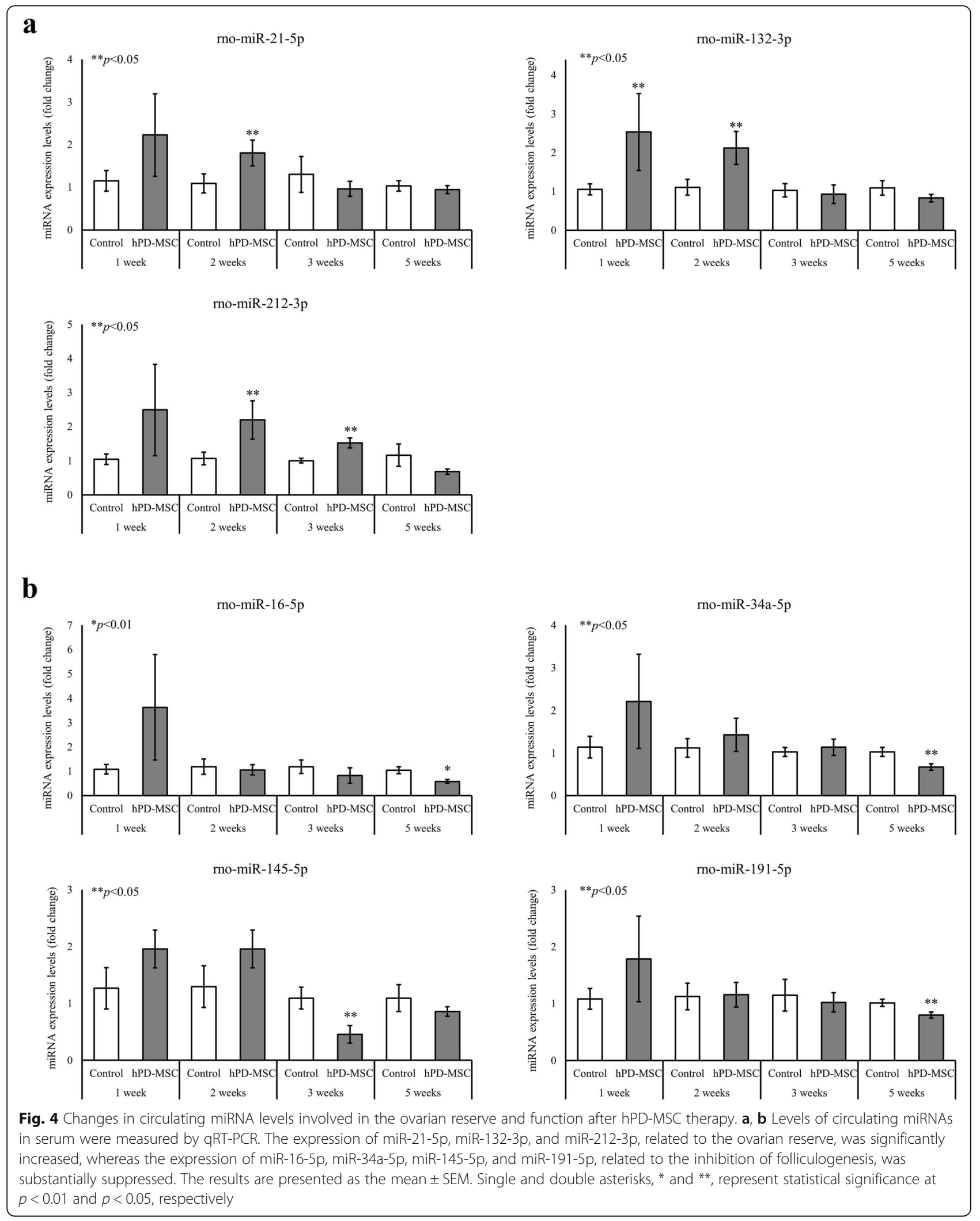




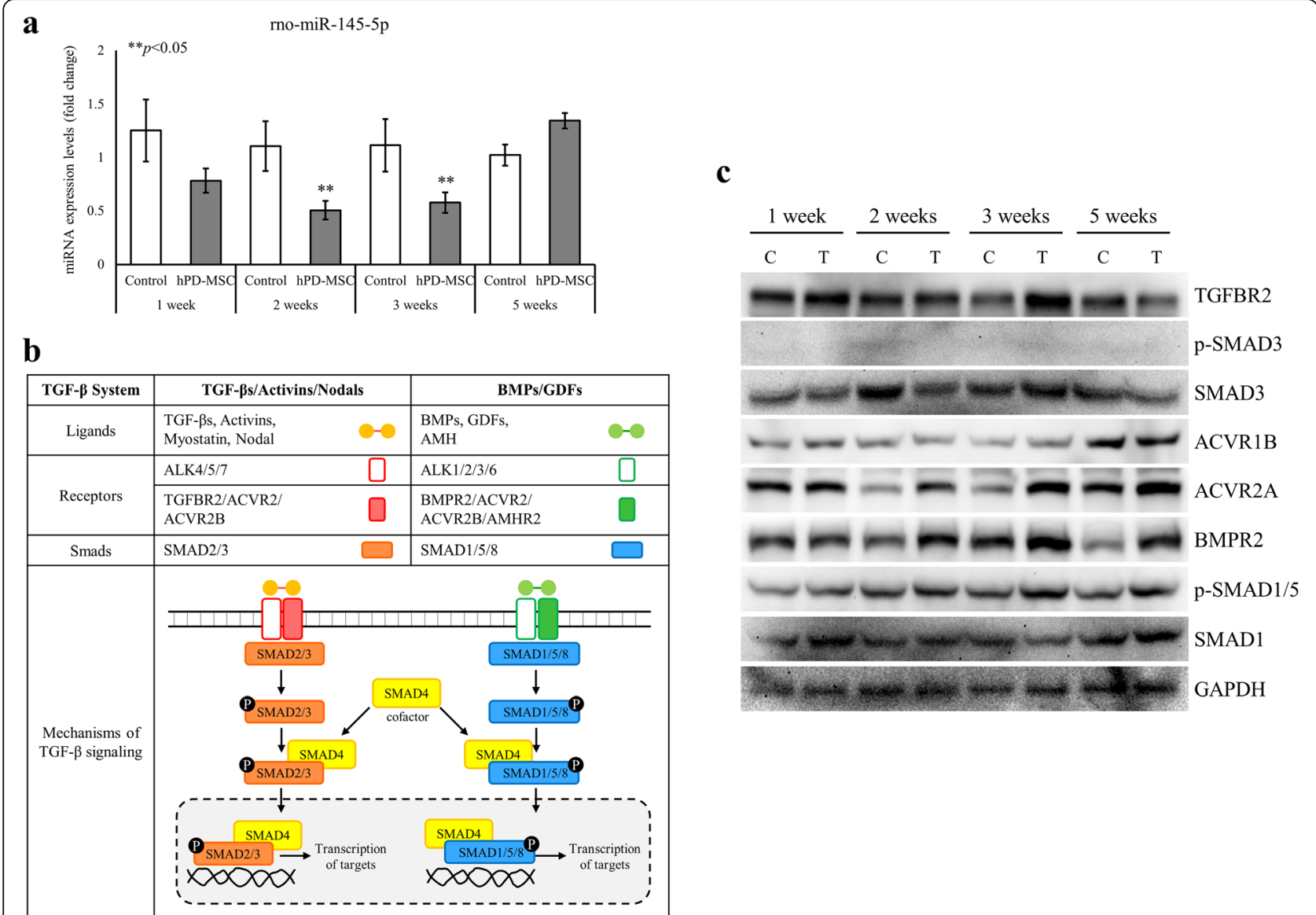

Fig. 5 Multiple-injection hPD-MSC therapy-induced miR-145-5p downregulation resulted in increased BMPR2 expression and SMAD pathway activation. a Levels of miR-145-5p in serum after multiple-injection therapy were measured by qRT-PCR. The expression of miR$145-5 p$ was significantly reduced after multiple-injection hPD-MSC therapy. The data are presented as the mean \pm SEM. The asterisk represents statistical significance at $p<0.05$. b Basic overview of two major SMAD pathways in TGF- $\beta$ signaling. TGF- $\beta$ s/Activins/Myostatin/ Nodal ligands bind to receptors and lead to the phosphorylation of SMAD2/3, which are translocated into the nucleus and subsequently activate the expression of target genes. However, BMPs/GDFs/AMH ligands activate receptors, phosphorylate SMAD1/5/8, and translocate to the nucleus. In the nucleus, p-SMAD1/5/8 proteins act as transcriptional cofactors that activate target gene expression. $\mathbf{c}$ Changes in the expression of proteins related to BMP and SMAD signaling in ovaries after the multiple-injection therapy. The expression of BMPR2 and p-SMAD1/5 was significantly increased after multiple-injection hPD-MSC therapy. GAPDH was used as a loading control. C, control group; T, multiple-injection therapy group

Likewise, p-SMAD1/5 expression was significantly increased in ovaries after multiple-injection hPD-MSC therapy (Fig. 5c). These findings strongly suggest that hPD-MSC therapy promotes the primordial-to-primary follicle transition through stem cell therapy-derived BMP signaling (Fig. 6).

\section{Discussion}

In the current study, the effects of single- and multipleinjection hPD-MSC therapy on ovarian aging were explored. hPD-MSCs were found in the ovaries after multiple-injection therapy and single-injection therapy, as we expected, and we observed attenuated ovarian dysfunction and increased ovarian reserve in old rat ovaries after hPD-MSC therapy. Importantly, our results showed that hPD-MSC transplantation through multiple injections dramatically increased the number of primary follicles through the primordial-to-primary follicle transition in aged ovaries, rescued hormone levels, and improved ovarian functions. The multiple-injection therapy changed the expression of circulating miRNAs and proteins associated with follicle development via BMP signaling and steroidogenesis in ovaries. In our experiments, compared to the single-injection therapy, the multiple-injection therapy was more effective in ovarian stimulation, increasing ovarian reserve, and functions, which suggested that multiple transplantations might be more valuable to clinically treat patients with ovarian dysfunction caused by aging.

Recently, because rodents are very useful models to study follicular development and ovarian function, it 


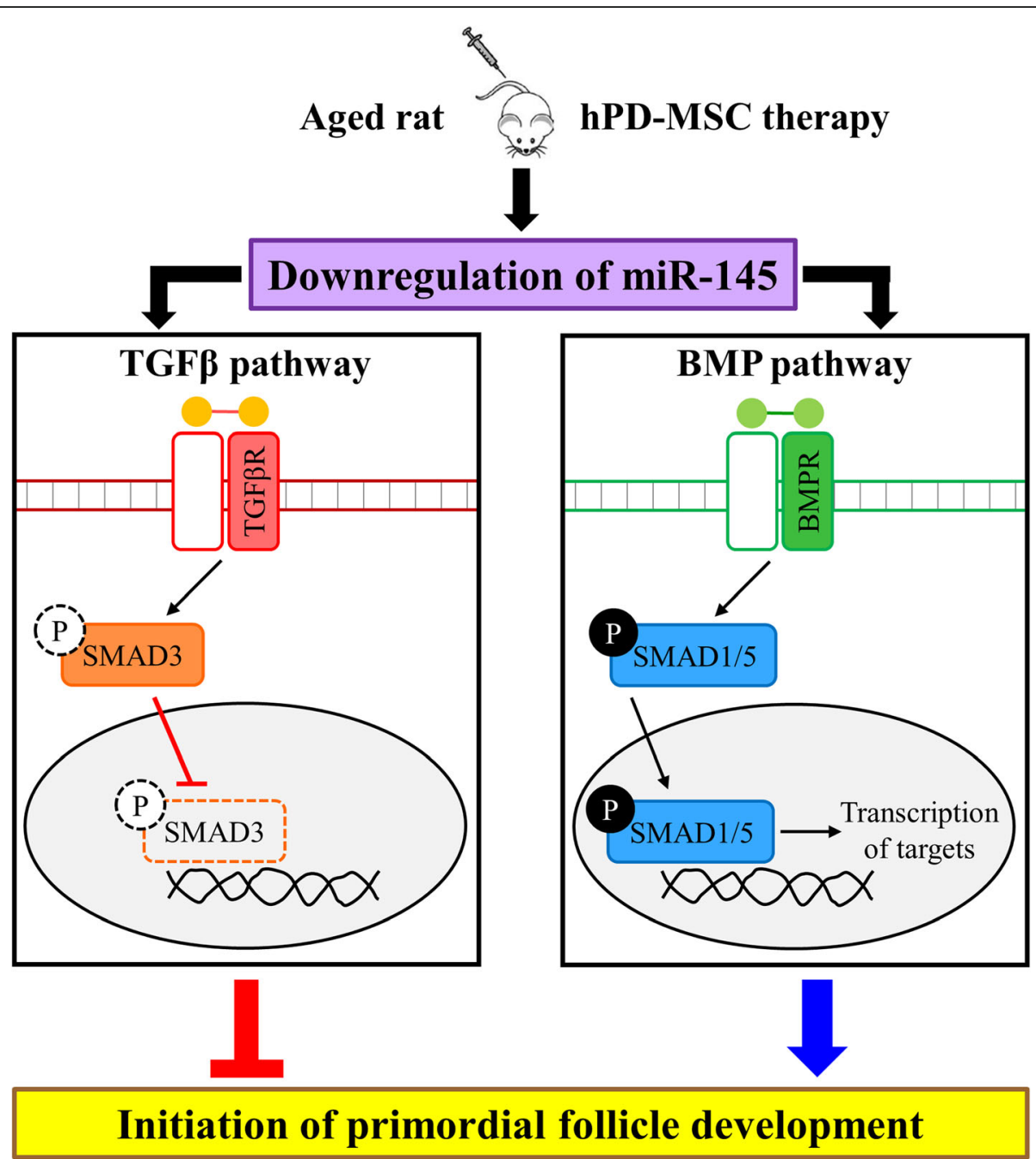

Fig. 6 Schematic diagram of the modulation of miR-145 expression and BMP-SMAD signaling by hPD-MSC therapies on folliculogenesis. When old female rats were treated with hPD-MSCs, there was a marked reduction in circulating miR-145 levels and restoration of BMP-SMAD1/5 signaling, but not of TGF- $\beta$ signaling, resulting in the activation of the primordial-to-primary follicle transition. As a result, aged ovaries had increased ovarian reserve and ovarian function

is important to know the similarities and differences in ovarian aging between rodents and humans. Previous work has established that SD rats at approximately 8-10 months of age undergo the reproductive aging process, showing decreased numbers of developing follicles and altered estrous cyclicity [40, 41]. In SD rats, a decline in fertility continues gradually until 12 months (approximately 52 weeks) of age [41]. From 12 months old onward, SD rats are infertile due to the absence of successful pregnancies and pups born per rat [42]. Therefore, for functional analysis after hPD-MSC therapy in ovaries with reproductive senescence, we used 12-month-old (52-54 weeks) female SD rats.

Menopause caused by aging and/or chemotherapy results in impaired hormone biosynthesis that causes metabolic syndrome, heart disease, depression, dementia, osteoporosis, atrophic vaginitis, and sexual dysfunction [4]. Recently, hormone replacement therapy has been the most effective treatment available for the disruptive symptoms of ovarian aging, which carries a risk for breast cancer and other diseases, including thromboembolism, stroke, vaginal bleeding, and heart disease [43, 44]. Previous studies established that mesenchymal stem cells (MSCs) can be used to treat animal models with chemotherapy- or OVX-induced early menopause as they ameliorate ovarian function by stimulating folliculogenesis [25, 45-47]. In this study, our results showed that the number of developing follicles and levels of hormones were significantly increased after hPD-MSC therapy in aged rats. After single- or multiple-injection therapy, hPD-MSCs did not induce xenogeneic immune responses in aged rats, indicating the low immunogenicity of 
hPD-MSCs. These results suggest that hPD-MSCs may represent an important cell source with clinical applications for improving ovarian function in older women.

The altered expression of miRNAs may affect folliculogenesis and ovarian steroidogenesis. Since the discovery that circulating miRNAs can be detected in different biofluids, including serum and follicular fluid, it has become evident that miRNAs can be used as novel biomarkers for the clinical diagnosis of ovarian health [20, 21, 48]. When rat ovaries with chemotherapy-induced damage were injected with stem cells stably expressing miR-21, improvements in ovarian structure and functions via the inhibition of granulosa cell apoptosis were observed [33]. Moreover, miR-132 upregulation in mouse granulosa cells promotes the production of $\mathrm{E}_{2}$ by the translational repression of Nurr1 [34]. In contrast, low expression of miR-16 and miR-191 in buffaloes is involved in ovarian follicular dominance and prevents ovarian follicular atresia [35]. In addition, downregulation of miR-34a and miR-145 resulted in suppression of granulosa cell apoptosis in a porcine model and activation of primordial follicle development in mice, respectively [32, 36]. Importantly, we showed that the levels of miR-21 and miR-132 were increased, whereas the expression of miR-16, miR-34a, miR145, and miR-191 was decreased after hPD-MSC therapy. These data indicate that hPD-MSC therapy leads to the altered expression of miRNAs related to folliculogenesis, hormone synthesis, granulosa cell apoptosis, and follicle atresia. Further studies examining the finely tuned miRNA-mediated regulation of ovarian function with aging after hPD-MSC therapy are needed.

The TGF- $\beta$ superfamily consists of secreted multifunctional proteins including TGF- $\beta$ s, AMH, activins, BMPs, and GDFs. These proteins are developmentally important growth factors that function via paracrine or autocrine signaling [49]. Currently, the TGF- $\beta$ superfamily is divided into two groups: TGF- $\beta$ s/activins and BMPs/GDFs [39]. TGF- $\beta$ superfamily ligands bind to serine/threonine kinase receptor type I (activin-like kinases; ALKs) and II (ACVR2A, ACVR2B, BMPR2, AMHR2, and TGFBR2) complexes on the cell surface that activate the SMAD pathway through the phosphorylation of SMAD proteins. Specifically, TGF- $\beta$ s, Activins, and Nodals are mediated by SMAD2 and SMAD3, while BMPs, GDFs, and AMH are mediated by SMAD1, SMAD5, and SMAD9. The phosphorylated SMAD proteins associate with SMAD4, translocate from the cytoplasm to the nucleus, and then regulate target gene transcription $[50,51]$. In the ovary, TGF- $\beta$ superfamily members are expressed in the oocyte, granulosa cells, and thecal cells at different follicle stages and contribute to the regulation of folliculogenesis [52]. In early folliculogenesis, TGF- $\beta$ s play pivotal roles in the maintenance of primordial follicle pools and in the activation of primordial follicles via SMAD-dependent and/or
SMAD-independent pathways [53, 54]. BMPs have also been implicated as positive intraovarian regulators of the primordial-to-primary follicle transition [38]. BMPR2 is expressed in the granulosa cells of primordial follicles in ruminants and in preantral follicles in rodents [55], which is in accordance with the high protein expression levels of BMPR2 in the ovaries after multiple-injection therapy in this study. In the present study, our results showed that SMAD1 and p-SMAD1/5 were highly expressed in ovaries after multiple-injection hPD-MSC therapy, suggesting that multiple-injection hPD-MSC therapy activated the classic BMP pathway characterized by SMAD1/5/8 signaling and activated the initiation of primordial follicle development in aged ovaries. Collectively, these results confirm the importance of hPD-MSC therapy-induced BMP-SMAD signaling in the primordial-to-primary follicle transition, which is critical for the ability to treat and/or attenuate female ovarian aging.

\section{Conclusions}

We demonstrated that the activation of the intraovarian BMP-SMAD1/5 signaling pathway by the downregulation of circulating miR-145 with stem cell therapy is essential for coordinating key events of the primordial-toprimary follicle transition in ovaries at advanced age (Fig. 6). Our results provide evidence that women with ovarian dysfunction accompanied by aging may have the option to use hPD-MSCs to rejuvenate their ovaries and to attenuate the effects of menopause via the return of ovarian function.

\section{Supplementary Information}

The online version contains supplementary material available at https://doi. org/10.1186/s13287-020-01988-X.

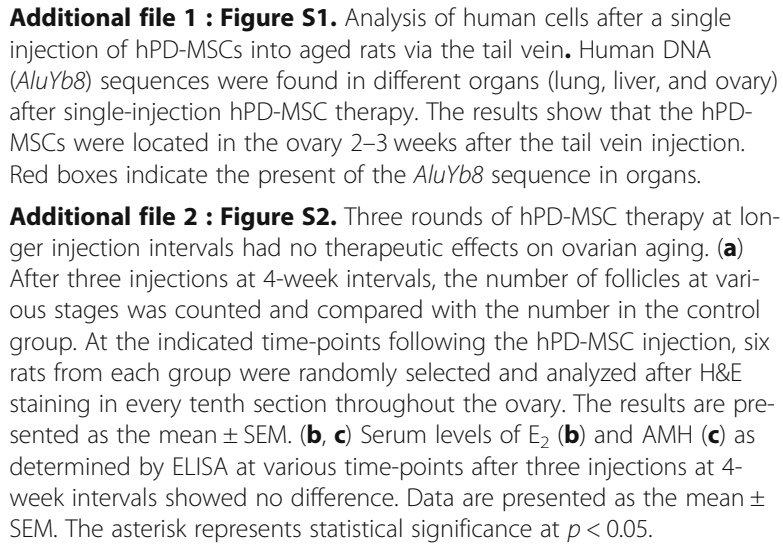

Additional file 1 : Figure S1. Analysis of human cells after a single injection of hPD-MSCs into aged rats via the tail vein. Human DNA (AluYb8) sequences were found in different organs (lung, liver, and ovary) after single-injection hPD-MSC therapy. The results show that the hPDMSCs were located in the ovary 2-3 weeks after the tail vein injection. Red boxes indicate the present of the AluYb8 sequence in organs.

Additional file $\mathbf{2}$ : Figure S2. Three rounds of hPD-MSC therapy at longer injection intervals had no therapeutic effects on ovarian aging. (a) After three injections at 4-week intervals, the number of follicles at various stages was counted and compared with the number in the control group. At the indicated time-points following the hPD-MSC injection, six rats from each group were randomly selected and analyzed after H\&E staining in every tenth section throughout the ovary. The results are presented as the mean \pm SEM. $(\mathbf{b}, \mathbf{c})$ Serum levels of $E_{2}(\mathbf{b})$ and $\mathrm{AMH}(\mathbf{c})$ as determined by ELISA at various time-points after three injections at 4week intervals showed no difference. Data are presented as the mean \pm SEM. The asterisk represents statistical significance at $p<0.05$.

Abbreviations

AMH: Anti-Müllerian hormone; BMP: Bone morphogenetic protein; $E_{2}$ : Estradiol; hPD-MSCs: Human placenta-derived mesenchymal stem cells; Ovx: Ovariectomy; TGF- $\beta$ : Transforming growth factor $\beta$ 


\section{Acknowledgements}

Not applicable.

\section{Authors' contributions}

K.-H.K., G.-J.K., M.-K.K., and K.-A.L. conceived and designed the experiments; K.-H.K. and E.-Y.K. performed the experiments; K.-H.K., J.J.K., K.-Y.C., and K.-A.L. analyzed the data; K.-H.K. and K.-A.L. wrote the manuscript; and K.-A.L. and G.-J.K. acquired financial support. All authors read and approved the final manuscript.

\section{Funding}

This research was supported by the Bio \& Medical Technology Development Program of the National Research Foundation and funded by the Korean government (NRF-2017M3A9B4061854).

\section{Availability of data and materials}

All data are included in the text and supplementary information.

\section{Ethics approval and consent to participate}

All procedures described herein were reviewed and approved by the Institutional Animal Care and Use Committee (IACUC) of CHA University and were performed in accordance with the Guiding Principles for the Care and Use of Laboratory Animals.

\section{Consent for publication}

Not applicable.

\section{Competing interests}

The authors declare that they have no competing interests.

\section{Author details}

Department of Biomedical Science, Institute of Reproductive Medicine, College of Life Science, CHA University, Pangyo-Ro 335, Bundang-gu, Seongnam-si, Gyeonggi-do 13488, South Korea. ${ }^{2}$ CHA Stem Cell Institute, CHA University, Pangyo-Ro 335, Bundang-gu, Seongnam-si, Gyeonggi-do 13488, South Korea. ${ }^{3} \mathrm{CHA}$ Fertility Center Seoul Station, CHA University School of Medicine, 416, Hangang-daero, Jung-gu, Seoul 04637, South Korea.

Received: 8 June 2020 Accepted: 20 October 2020

Published online: 05 November 2020

\section{References}

1. Amanvermez $R$, Tosun M. An update on ovarian aging and ovarian reserve tests. Int J Fertil Steril. 2016:9:411-5.

2. Tatone C, Amicarelli F, Carbone MC, Monteleone P, Caserta D, Marci R, et al. Cellular and molecular aspects of ovarian follicle ageing. Hum Reprod Update. 2008;14:131-42.

3. Broekmans FJ, Soules MR, Fauser BC. Ovarian aging: mechanisms and clinical consequences. Endocr Rev. 2009;30:465-93.

4. Faubion SS, Kuhle CL, Shuster LT, Rocca WA. Long-term health consequences of premature or early menopause and considerations for management. Climacteric. 2015;18:483-91.

5. Faddy MJ, Gosden RG. A model conforming the decline in follicle numbers to the age of menopause in women. Hum Reprod. 1996;11:1484-6.

6. Borum K. Oogenesis in the mouse. A study of the meiotic prophase. Exp Cell Res. 1961;24:495-507.

7. Adhikari D, Liu K. Molecular mechanisms underlying the activation of mammalian primordial follicles. Endocr Rev. 2009;30:438-64.

8. Hansen KR, Knowlton NS, Thyer AC, Charleston JS, Soules MR, Klein NA. A new model of reproductive aging: the decline in ovarian non-growing follicle number from birth to menopause. Hum Reprod. 2008;23:699-708.

9. Hsueh AJ, Kawamura K, Cheng Y, Fauser BC. Intraovarian control of early folliculogenesis. Endocr Rev. 2015;36:1-24

10. Hutt KJ, McLaughlin EA, Holland MK. KIT/KIT ligand in mammalian oogenesis and folliculogenesis: roles in rabbit and murine ovarian follicle activation and oocyte growth. Biol Reprod. 2006:75:421-33.

11. Roberts AE, Arbogast LK, Friedman Cl, Cohn DE, Kaumaya PT, Danforth DR Neutralization of endogenous vascular endothelial growth factor depletes primordial follicles in the mouse ovary. Biol Reprod. 2007;76:218-23.
12. Tanwar PS, O'Shea T, McFarlane JR. In vivo evidence of role of bone morphogenetic protein-4 in the mouse ovary. Anim Reprod Sci. 2008;106: 232-40.

13. Lee WS, Otsuka F, Moore RK, Shimasaki S. Effect of bone morphogenetic protein-7 on folliculogenesis and ovulation in the rat. Biol Reprod. 2001;65: 994-9.

14. Nilsson EE, Kezele P, Skinner MK. Leukemia inhibitory factor (LIF) promotes the primordial to primary follicle transition in rat ovaries. Mol Cell Endocrinol. 2002:188:65-73.

15. Nilsson E, Parrott JA, Skinner MK. Basic fibroblast growth factor induces primordial follicle development and initiates folliculogenesis. Mol Cell Endocrinol. 2001;175:123-30.

16. Oktem O, Urman B. Understanding follicle growth in vivo. Hum Reprod. 2010;25:2944-54.

17. Findlay JK, Hutt KJ, Hickey M, Anderson RA. How is the number of primordial follicles in the ovarian reserve established? Biol Reprod. 2015;93: 111.

18. Ambros $V$. The functions of animal microRNAs. Nature. 2004;431:350-5.

19. Jung HJ, Suh Y. Circulating miRNAs in ageing and ageing-related diseases. J Genet Genomics. 2014;41:465-72.

20. Yang $X$, Zhou Y, Peng S, Wu L, Lin HY, Wang S, et al. Differentially expressed plasma microRNAs in premature ovarian failure patients and the potential regulatory function of mir-23a in granulosa cell apoptosis. Reproduction. 2012;144:235-44.

21. Imbar T, Eisenberg I. Regulatory role of microRNAs in ovarian function. Fertil Steril. 2014:101:1524-30.

22. Lee MJ, Jung J, Na KH, Moon JS, Lee HJ, Kim JH, et al. Anti-fibrotic effect of chorionic plate-derived mesenchymal stem cells isolated from human placenta in a rat model of CCl(4)-injured liver: potential application to the treatment of hepatic diseases. J Cell Biochem. 2010;111:1453-63.

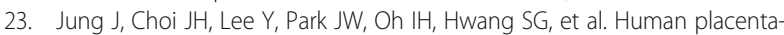
derived mesenchymal stem cells promote hepatic regeneration in $\mathrm{CCl} 4$ -injured rat liver model via increased autophagic mechanism. Stem Cells. 2013:31:1584-96.

24. Abumaree $\mathrm{MH}$, Al Jumah MA, Kalionis B, Jawdat D, Al Khaldi A, Abomaray FM, et al. Human placental mesenchymal stem cells (pMSCs) play a role as immune suppressive cells by shifting macrophage differentiation from inflammatory M1 to anti-inflammatory M2 macrophages. Stem Cell Rev Rep. 2013:9:620-41.

25. Kim TH, Choi JH, Jun Y, Lim SM, Park S, Paek JY, et al. 3D-cultured human placenta-derived mesenchymal stem cell spheroids enhance ovary function by inducing folliculogenesis. Sci Rep. 2018;8:15313.

26. Triant DA, Whitehead A. Simultaneous extraction of high-quality RNA and DNA from small tissue samples. J Hered. 2009;100:246-50.

27. Walker JA, Kilroy GE, Xing J, Shewale J, Sinha SK, Batzer MA. Human DNA quantitation using Alu element-based polymerase chain reaction. Anal Biochem. 2003:315:122-8.

28. Kim KH, Kim EY, Lee SY, Ko JJ, Lee KA. Oocyte cytoplasmic Gas6 and heparan sulfate (HS) are required to establish the open chromatin state in nuclei during remodeling and reprogramming. Cell Physiol Biochem. 2018; 45:37-53.

29. Agoston DV. How to translate time? The temporal aspect of human and rodent biology. Front Neurol. 2017:8:92

30. Sengupta P. The laboratory rat: relating its age with human's. Int J Prev Med. 2013:4:624-30.

31. Sorensen AE, Wissing ML, Englund AL, Dalgaard LT. MicroRNA species in follicular fluid associating with polycystic ovary syndrome and related intermediary phenotypes. J Clin Endocrinol Metab. 2016;101:1579-89.

32. Yang $S$, Wang $S$, Luo A, Ding T, Lai Z, Shen W, et al. Expression patterns and regulatory functions of microRNAs during the initiation of primordial follicle development in the neonatal mouse ovary. Biol Reprod. 2013;89:126.

33. Fu $X$, He $Y$, Wang $X$, Peng $D$, Chen $X$, Li $X$, et al. Overexpression of miR-21 in stem cells improves ovarian structure and function in rats with chemotherapy-induced ovarian damage by targeting PDCD4 and PTEN to inhibit granulosa cell apoptosis. Stem Cell Res Ther. 2017;8:187.

34. Wu S, Sun H, Zhang Q, Jiang Y, Fang T, Cui I, et al. MicroRNA-132 promotes estradiol synthesis in ovarian granulosa cells via translational repression of Nurr1. Reprod Biol Endocrinol. 2015:13:94

35. Singh P, Golla N, Singh P, Baddela VS, Chand S, Baithalu RK, et al. Salivary miR-16, miR-191 and miR-223: intuitive indicators of dominant ovarian follicles in buffaloes. Mol Gen Genomics. 2017;292:935-53. 
36. Tu F, Pan ZX, Yao Y, Liu HL, Liu SR, Xie Z, et al. miR-34a targets the inhibin beta $\mathrm{B}$ gene, promoting granulosa cell apoptosis in the porcine ovary. Genet Mol Res. 2014;13:2504-12.

37. Hu Z, Shen WJ, Kraemer FB, Azhar S. Regulation of adrenal and ovarian steroidogenesis by miR-132. J Mol Endocrinol. 2017;59:269-83.

38. Knight PG, Glister C. TGF-beta superfamily members and ovarian follicle development. Reproduction. 2006;132:191-206.

39. Trombly DJ, Woodruff TK, Mayo KE. Roles for transforming growth factor beta superfamily proteins in early folliculogenesis. Semin Reprod Med. 2009; 27:14-23.

40. Peng MT, Huang $\mathrm{HH}$. Aging of hypothalamic-pituitary-ovarian function in the rat. Fertil Steril. 1972;23:535-42.

41. Acuna E, Fornes R, Fernandois D, Garrido MP, Greiner M, Lara HE, et al. Increases in norepinephrine release and ovarian cyst formation during ageing in the rat. Reprod Biol Endocrinol. 2009;7:64.

42. Cruz G, Fernandois D, Paredes AH. Ovarian function and reproductive senescence in the rat: role of ovarian sympathetic innervation. Reproduction. 2017;153:R59-68.

43. Xu L. How to judge the association of postmenopausal hormone therapy and the risk of breast cancer. Front Med China. 2010;4:290-3.

44. Kwak EK, Park HS, Kang NM. Menopause knowledge, attitude, symptom and management among midlife employed women. J Menopausal Med. 2014; 20:118-25.

45. Ling $L$, Feng $X$, Wei $T$, Wang $Y$, Wang $Y$, Zhang $W$, et al. Effects of lowintensity pulsed ultrasound (LIPUS)-pretreated human amnion-derived mesenchymal stem cell (hAD-MSC) transplantation on primary ovarian insufficiency in rats. Stem Cell Res Ther. 2017;8:283.

46. Fu X, He Y, Xie C, Liu W. Bone marrow mesenchymal stem cell transplantation improves ovarian function and structure in rats with chemotherapy-induced ovarian damage. Cytotherapy. 2008;10:353-63.

47. Zhu SF, Hu HB, Xu HY, Fu XF, Peng DX, Su WY, et al. Human umbilical cord mesenchymal stem cell transplantation restores damaged ovaries. J Cell Mol Med. 2015;19:2108-17.

48. Li Y, Fang $Y$, Liu Y, Yang $X$. MicroRNAs in ovarian function and disorders. J Ovarian Res. 2015:8:51

49. Ingman WV, Robertson SA. Defining the actions of transforming growth factor beta in reproduction. Bioessays. 2002:24:904-14

50. Drummond AE. TGFbeta signalling in the development of ovarian function. Cell Tissue Res. 2005:322:107-15.

51. Heldin CH, Miyazono K, ten Dijke P. TGF-beta signalling from cell membrane to nucleus through SMAD proteins. Nature. 1997;390:465-71.

52. Kaivo-oja N, Jeffery LA, Ritvos O, Mottershead DG. Smad signalling in the ovary. Reprod Biol Endocrinol. 2006:4:21.

53. Wang ZP, Mu XY, Guo M, Wang YJ, Teng Z, Mao GP, et al. Transforming growth factor-beta signaling participates in the maintenance of the primordial follicle pool in the mouse ovary. J Biol Chem. 2014;289:8299-311.

54. Granados-Aparici S, Hardy K, Franks S, Sharum IB, Waite SL, Fenwick MA. SMAD3 directly regulates cell cycle genes to maintain arrest in granulosa cells of mouse primordial follicles. Sci Rep. 2019;9:6513.

55. Edwards SJ, Reader KL, Lun S, Western A, Lawrence S, McNatty KP, et al. The cooperative effect of growth and differentiation factor-9 and bone morphogenetic protein (BMP)-15 on granulosa cell function is modulated primarily through BMP receptor II. Endocrinology. 2008;149:1026-30.

\section{Publisher's Note}

Springer Nature remains neutral with regard to jurisdictional claims in published maps and institutional affiliations.

Ready to submit your research? Choose BMC and benefit from:

- fast, convenient online submission

- thorough peer review by experienced researchers in your field

- rapid publication on acceptance

- support for research data, including large and complex data types

- gold Open Access which fosters wider collaboration and increased citations

- maximum visibility for your research: over $100 \mathrm{M}$ website views per year

At $\mathrm{BMC}$, research is always in progress.

Learn more biomedcentral.com/submissions 